\title{
THE THIRTY-THIRD ANNUAL MEETING OF THE SOCIETY
}

The thirty-third Annual Meeting of the American Mathematical Society was held at the University of Pennsylvania, from Tuesday to Thursday, December 28-30, 1926, inclusive, in conjunction with the meetings of the Mathematical Association of America and the American Association for the Advancement of Science. The mornings and afternoons of Tuesday and Wednesday were devoted to the regular scientific sessions of the Society; the morning sessions were in sections, on Tuesday (i) Applications and Analysis, and (ii) Geometry, and on Wednesday (i) Analysis and (ii) Point Sets and Geometry. The Gibbs Lecture was delivered on Tuesday afternoon. On Thursday morning a joint session of the two mathematical organizations and Section A of the American Association for the Advancement of Science was held. The joint dinner on Wednesday evening at the Aldine Hotel was attended by about one hundred seventy-five mathematicians and their friends; Professor E. V. Huntington, Vice-President of Section A, presided.

At the joint session on Thursday morning, it was voted to express to the University of Pennsylvania and its Department of Mathematics and also to the Drexel Institute and other inviting organizations the thanks of the Society for their hospitality during the meeting, the largest in the history of the Society.

The attendance included the following two hundred four members of the Society:

C. R. Adams, Archibald, Atchison, W. L. Ayres, R. W. Babcock, Bacon, Barney, F. W. Beal, A. A. Bennett, Benton, William James Berry, Bill, Birkhoff, G. A. Bliss, Blumberg, Bower, Bradshaw, Brasefield, B. H. Brown, H. S. Brown, Margaret Buchanan, R. W. Burgess, Caldwell, W. B. Campbell, Caris, Carlen, Chapelon, Clawson, Clutz, Coble, Abraham Cohen, Coleman, J. T. Colpitts, Comegys, Crane, Crawley, Currier, Curry, Curtiss, Dadourian, Daniells, J. E. Davis, Decker, Dederick, Dillingham, Dimick, Douglas, Edmondson, Eiesland, Eisenhart, 
W. W. Elliott, Eshleman, H. B. Evans, Everett, Feinler, Finkel, Fite, Focke, Foraker, W. B. Ford, C. H. Forsyth, Fort, M. C. Foster, Philip Franklin, Fry, C. A. Garabedian, Garretson, Gehman, D. C. Gillespie, Gleason, Glenn, Gravatt, C. C. Grove, Guggenbühl, Hallett, Hancock, M. G. Haseman, Haskins, Hazlett, Hedlund, E. R. Hedrick, H. B. Hedrick, Archibald Henderson, Robert Henderson, Horton, Hosford, Huber, J. C. Hughes, Huntington, Hurwitz, F. K. Hyatt, Louis Ingold, Ingraham, Dunham Jackson, M. I. Johnson, R. A. Johnson, O. D. Kellogg, Knebelman, Kormes, Lambert, Lamond, Langer, Langford, Lefschetz, Lehr, Harry Levy, F. P. Lewis, Littauer, Locke, Logsdon, McGiffert, McMackin, MacColl, MacInnes, E. N. Martin, Mears, Meder, Miser, H. H. Mitchell, Molina, R. L. Moore, Richard Morris, Marston Morse, Murnaghan, F. H. Murray, Nassau, Neelley, Olds, F. W. Owens, H. B. Owens, B. C. Patterson, Paxton, Pehrson, Pepper, E. C. Phillips, Pierpont, Polley, R. G. Putnam, Ragsdale, Rau, Raynor, C. J. Rees, Reid, C. N. Reynolds, J. N. Rice, R. G. D. Richardson, H. L. Rietz, Ritt, E. D. Roe, Roever, Root, Rorer, Rowe, Rowland, Safford, Sakellariou, Seely, Seidlin, Shewhart, Shugert, Silverman, W. G. Simon, L. G. Simons, Slaught, Smail, A. W. Smith, W. M. Smith, Virgil Snyder, Starke, Stokes, Swartzel, Swift, Tamarkin, Thurston, Torrey, B. M. Turner, Tyler, Uhler, Vandiver, Veblen, Vivian, Waddell, G. W. Walker, J. L. Walsh, Wedderburn, Weida, V. H. Wells, A. P. Wheeler, A. M. Whelan, R. A. Whelan, Whited, Widder, C. E. Wilder, F. B. Williams, K. P, Williams, W. L. G. Williams, A. H. Wilson, E. W. Wilson, R. G. Wood, Wyant, Yeaton, J. W. Young, M. M. Young, Zippin.

The Council elected the following sixteen persons to membership in the Society:

Mr. Ross Harvey Bardell, University of South Dakota;

Professor Stanley Eugene Brasefield, Rutgers University;

Professor Ona Kenneth DeFoe, College of the Ozarks;

Mr. Victor C. d'Unger, Little Rock, Arkansas;

Mr. Aurelius Augustus Evans, Columbia University;

Professor Hemphill Moffett Hosford, Southern Methodist University;

Miss Olive Allan Kee, Teachers College, Boston;

Mr. Eugene A. Kholodovsky, Columbia University;

Mr. Edward Dennis McCarthy, Pennsylvania State College;

Mr. Guerdon David Nichols, Colorado School of Mines;

Miss Katharine Elizabeth O'Brien, College of New Rochelle;

Mr. Thomas Howard Rawles, Yale University;

Mr. George Wesley Riddle, Lehigh University;

Mr. Homer Morgan Rutherford, Houston, Texas;

Mr. Albert Edward Staniland, University of Pittsburgh;

Professor William John Webber, University of Toronto.

The following four persons were elected to membership as nominees of Allyn and Bacon: 
Mr. H. V. Craig, University of Wisconsin;

Mr. Lawrence Sanford Kennison, Brown University;

Miss Rose Alice Whelan, Bryn Mawr College;

Mr. Arthur Simeon Winsor, Johns Hopkins University.

The following three persons were elected to membership as nominees of the National Life Insurance Company of the United States of America:

Mr. George Cramer, University of Missouri;

Miss Florence Marie Mears, Cornell University;

Mr. Aubrey Henderson Smith, Brown University.

As a nominee of the University of Pennsylvania was elected:

Mr. Leo Zippin.

The ordinary membership in the Society is now 1692, including 178 nominees of sustaining members and 82 life members. There are also 38 sustaining members. The total attendance of members at all meetings during the past year was 729 ; the number of papers read was 361 . The number of members attending at least one meeting was 438 . At the annual election 275 votes were cast.

The reports of the Treasurer and of the auditors (Mr. S. A. Joffe and Professor H. W. Reddick) were received, showing a balance of $\$ 4214.93$, exclusive of the balances in the Bulletin, Transactions, Colloquium and special funds, and in the life membership reserve. A vote of thanks was tendered Mr. Joffe for his splendid services extending over several years.

The Society's Endowment Fund now has securities of par value $\$ 64,000$, yielding an annual income of $\$ 2975$; sustaining membership fees for the year amounted to $\$ 5000$. The amount received from sales of the Society's publications was $\$ 4849.30$.

The Board of Trustees adopted a budget for 1927 showing expenditures and receipts as $\$ 31,898.56$ and $\$ 29,473.56$ respectively. The deficit for the year can be met from the balance brought forward from 1926.

The Librarian reported that the Library of the Society now contains 7239 volumes. A catalogue of the Library was printed and distributed early in 1926. 
At the annual election, which closed on Tuesday afternoon, the following trustees and officers and other members of the Council were chosen:

Board of Trustees, Professor W. B. Fite, Mr. Robert Henderson, Professors R. G. D. Richardson, Virgil Snyder, Oswald Veblen.

President, Professor Virgil Snyder.

Vice-President, Professor O. D. Kellogg.

Assistant Secretary, Professor Arnold Dresden.

Librarian, Professor R. C. Archibald.

Member of the Editorial Committee of the Bulletin, Professor Arnold Dresden.

Member of the Editorial Committee of the Transactions, Professor Edward Kasner.

Members of the Council: for one year, Professor Daniel Buchanan; for three years, Professors A. A. Bennett, J. W. Glover, James Pierpont, H. S. Vandiver, G. E. Wahlin.

The tellers appointed by President Birkhoff to count the ballots were Professors C. R. Adams and R. G. Putnam.

The following appointments were reported: to represent the Society on the Sectional Committee on Standards for Graphic Presentation sponsored by the American Society of Mechanical Engineers, Professor W. H. Roever; to represent the Society at the American Mining Congress to be held in Washington, D. C., December 7-10, 1927, Mr. C. E. Van Orstrand, of the Geological Survey; as Committee on Arrangements for the Annual Meeting of 1927 in Nashville, Professors C. M. Sarratt (chairman), W. L. Miser, G. D. Evans, Archibald Henderson, Arnold Dresden, and R. G. C. Richardson; to represent the Society in collaboration with the History of Science Society, the American Physical Society, and the American Astronomical Society, in the celebration in New York City, during the coming spring, of the bicentenary of Newton's death, Professors R. C. Archibald and E. W. Brown.

An account of the agreement between the Society and the Johns Hopkins University regarding the conduct of the 
American Journal of Mathematics appears on page 1 of the January-February issue of this Bulletin.

At the meeting of the Council, Professor G. C. Evans was named to succeed Professor H. F. Blichfeldt as representative of the Society in the National Research Council. Professors Arnold Dresden and Tomlinson Fort were appointed to represent the Society on the Council of the American Association for the Advancement of Science for the year 1927. As the Committee on the Award of the Frank Nelson Cole Prize, which will take place at the Annual Meeting of 1927, the following were appointed: Professors H. H. Mitchell (chairman), E. T. Bell, and H. F. Blichfeldt.

At the request of Professor J. H. M. Wedderburn, the Society named three associate editors on the board of the Annals of Mathematics for a period of three years. These persons are: Professors D. C. Gillespie, W. L. Hart, and R. E. Langer.

It was reported that the Committee on Colloquia had accepted for the Colloquium Series a book by Professor G. C. Evans entitled Potential Theory.

It was reported that Professor Coble has accepted the invitation of the Council to give a colloquium lecture at the summer meeting of 1928 at Amherst, and that his subject is The space sextic of genus four. Professor R. L. Moore was invited to give a colloquium lecture at the Boulder meeting in the summer of 1929.

The fourth Josiah Willard Gibbs Lecture was delivered on Tuesday afternoon by Dr. H. B. Williams, Dalton professor of Physiology at Columbia University. This lecture, which will appear in full in the May-June issue of this Bulletin, was entitled Mathematics and the biological sciences. The fifth Josiah Willard Gibbs Lecture will be given at Nashville in December, 1927. Professors Tomlinson Fort (chairman), O. D. Kellogg, and M. B. Porter were appointed to recommend to the Council a lecturer. 
At the joint session of the Society, the Mathematical Association, and Section A, held on Thursday morning, the following papers were read:

I. A mathematical critique of some physical theories, by Professor G. D. Birkhoff, retiring President of the Society. This paper appears in full in the present issue of this Bulletin.

II. The weight field of force of the earth, by Professor W. H. Roever, retiring Vice-President of Section A.

III. The duty of exposition (with special reference to the Cauchy-Heaviside expansion theorem), by Professor F. D. Murnaghan. (Address delivered at the request of the Mathematical Association of America.)

An event of the highest significance was the award to President Birkhoff, for his retiring address, of the fourth American Association for the Advancement of Science Prize of one thousand dollars. This prize is awarded to some person presenting at the annual meeting a notable contribution to the advancement of science. It will be recalled that the first prize was awarded to Professor L. E. Dickson three years ago.

Titles and abstracts of the papers read at the regular sessions of the Society follow below. On Tuesday morning, Professor J. H. M. Wedderburn presided over the Section of Applications and Analysis, relieved by Professor H. H. Mitchell, and Professor F. D. Murnaghan over the Section of Geometry; retiring President Birkhoff presided on Tuesday afternoon. Professor Marston Morse presided over the Section of Analysis on Wednesday morning, and Professor R. L. Moore over that of Point Sets and Algebra; President Virgil Snyder presided on Wednesday afternoon. Professor Sibert's paper was communicated by Professor Hancock. The papers of Blumberg (first paper), B. H. Camp, C. C. Camp, Evans, Fort, Franklin (first paper), Glenn, Graustein, Hazlett (second paper), Hollcroft, Koopman, Pierpont (first paper), Schwatt, Vandiver (first paper), Wells, Widder (second and third papers), Wilder, and Zeldin were read by 
title. Dr. Mandelbrojt was introduced by Professor Evans, and Professor Sibert by Professor Hancock.

1. Professor C. H. Forsyth: The yield of a dividend paying venture.

The author offers a solution of the oft occurring problem of finding the yield of property, bought at one price and sold at another, taking into consideration any regular dividend. Two rates of interest are used, in addition to the dividend rate, to give a direct solution, not an approximated result. The solution applies as well, of course, to deals in stocks and even in bonds.

2. Professor J. R. Musselman: On the linear correlation ratio in the case of certain symmetrical frequency distributions.

The author has extended the idea of a linear correlation ratio, which is of use when one of the characters is in broad categories, to the frequency surface, with linear regression, for which the $\beta_{1}$ 's in the case of both marginal totals are zero, and the $\beta_{2}$ 's are equal but arbitrary. This article has been accepted by Biometrika.

3. Professor C. A. Garabedian: Correction of certain results on the flexure of a thick circular plate given by de Saint-Venant in the celebrated "Note finale $d u$ \$45" of the translation of Clebsch.

In this examination of de Saint-Venant's note on a method for dealing with the flexure of a thick circular plate (1883), it is shown that all the solutions obtained are in error in so far as terms of the second order are concerned. The suppression of these terms would reduce the formulas to the thin plate solutions given by Poisson in the memoir of 1828. Although de Saint-Venant's results were questioned by Karl Pearson in 1893 (History of the Theory of Elasticity, vol. 2, part 1), they continued to be accepted, and are still to be found in literature of the present day. By extension of methods of analysis developed in the original note, the present paper obtains the correct solutions, and thus removes certain contradictions that de Saint-Venant attempted to explain away by a physical argument.

4. Professor C. R. Adams: On the series formally satisfying a difference equation whose characteristic equation has irregular roots.

A root of the characteristic equation is said to be regular when it is simple, finite, and non-zero. In the present paper formal series are found for the linear difference equation of $n$th order with rational coefficients in all possible irregular cases subject only to the restriction that when a root is multiple a certain secondary condition is not satisfied. When multiple roots occur, the series are in general analogous to the anormal series of differential equations; i.e., to a root of multiplicity $m$ there correspond in 
general $m$ series in $1 / x^{1 / m}$, multiplied by suitable factors. These results extend considerably beyond those previously obtained in this field, which are limited to the case in which the only irregularity is one zero root (Horn, 1910) and to the irregular cases of a second-order equation (Batchelder, 1913).

\section{Professor C. R. Adams: Analytic solutions of the linear difference equation in the irregular case.}

The formal series found in the preceding paper are here made the basis for a study of the existence and properties of analytic solutions; the general method employed is that used by Birkhoff in the regular case. The coefficients being assumed rational, we show that in many cases there exist two sets of $n$ solutions each. In most of these cases we find that one solution from each set is meromorphic over the entire finite plane, and is asymptotically represented in a certain sector $(>\pi)$ by one of the formal series; the remaining $n-1$ solutions of each set are meromorphic in the portion of the plane above (or below) some parallel to the axis of reals and are represented asymptotically by the remaining $n-1$ formal series in the sector $\epsilon \leqq \arg x \leqq \pi-\epsilon$ (or $-\pi+\epsilon \leqq \arg x \leqq-\epsilon), \epsilon$ being an arbitrarily small positive number. In certain cases, however, the situation is more like the regular case, in that there exist two full sets of solutions which are meromorphic over the entire finite plane and asymptotically represented, in certain sectors, by the formal series.

\section{Professor R. E. Langer: Three theorems on closure of biorthogonal systems of functions.}

This paper has appeared in the January-February issue of this Bulletin.

7. Professor R. E. Langer and Mrs. Eleanor P. Brown: On the theory of integral equations with discontinuous kernels.

In an earlier paper by one of the authors, the theory of the integral equation $u(x)=\lambda \int_{a}^{b} K(x, \xi) u(\xi) d \xi$, with a kernel which is discontinuous along $\xi=x$, was developed. The present paper considers the case in which the kernel is continuous while a first partial derivative possesses a finite non-vanishing discontinuity along $\xi=x$. As in the earlier paper, the method used centers on a transformation of the given equation into an integrodifferential system. It is proved under hypotheses of a general nature that there exist infinitely many characteristic values of the parameter $\lambda$, and asymptotic expressions for these values and for the characteristic. functions are obtained. The closure of the set of solutions is established, and the problem of the expansion of an arbitrary function in a series of solutions is discussed.

8. Professors R. E. Langer and J. D. Tamarkin: On the theory of integral equations with discontinuous kernels.

This paper deals with the integral equation $u(x)=\lambda \int_{a}^{b} K(x, \xi) u(\xi) d \xi$, whose kernel $K(x, \xi)$ possesses partial derivatives of order $n$, which are 
discontinuous along the line $\xi=x$, while the kernel itself and its partial derivatives of order $(n-1)$ are continuous. Under suitable restrictions of more or less general character, the existence of infinitely many characteristic values is established, and asymptotic expressions for these values and for the characteristic functions are found. A theorem on the expansion of an arbitrary function in a series of characteristic functions is proved. The method used is a combination of methods used by the authors individually in previous papers on integral and integro-differential equations.

9. Professor J. D. Tamarkin: The notion of the Green's function in the theory of integro-differential equations. Second paper.

The subject of this paper is the integro-differential boundary problem $u^{(n)}+p_{1}(x, \rho) u^{(n-1)}+\cdots+p_{n}(x, \rho) u=f(x)+\int_{a}^{b} H(x, \xi, \rho) u(\xi) d \xi ; L_{i}(u)=$ $0, i=1,2, \cdots, n$, where $p_{i}(x, \rho)$ is a polynomial in $\rho$ of the $i$ th degree and $H(x, \xi, \rho)$ is of the $n$th degree in $\rho$. The operators $L_{i}(u)$ are of the type involving integrals of $u$ as well as the functional values at the end points. The case in which $H(x, \xi, \rho)$ is of the $(n-1)$ th degree in $\rho$ has been treated in an earlier paper by the author. The discussion in the present paper is based upon an elementary lemma of the theory of the Fredholm integral equation, which generalizes known properties of orthogonal kernels. All the results of the first paper concerning the existence and asymptotic expressions of characteristic values and the equiconvergence theorem are extended to the case in hand. The equiconvergence theorem is not valid unless the usual Birkhoff integral is replaced by a more complicated one.

\section{Dr. B. O. Koopman: On multiform integrals of differ-} ential equations. Preliminary report.

Consider the system of equations $d x_{1} / X_{1}=\cdots=d x_{n} / X_{n}$, either in the real or the complex domain. The $X$ 's are single-valued analytic functions of the $x$ 's, nowhere all zero, in a certain region $R$. Suppose that the allied partial differential equation (1): $X_{1}\left(\partial f / \partial x_{1}\right)+\cdots+X_{n}\left(\partial f / \partial x_{n}\right)=0$ admits a system of integrals $f_{1}, \cdots, f_{n-1}$, each of which allows of indefinite analytic continuation throughout $R$, and suppose that at every point of $R$ the $f$ 's form an independent system. If we start from a point $P$ with a given determination of $f_{1}, \cdots, f_{n-1}$, and continue the functions analytically back to $P$ along a loop $\Gamma$ which cannot be shrunk to a point in the region $R$, we shall obtain a second system of branches, $f_{1}{ }^{\prime}, \cdots, f_{n}{ }^{\prime}$, in general distinct from the first. But since there can be no more than $n-1$ independent integrals of (1) at $P$, we shall have (2): $f_{k}{ }^{\prime}=\theta_{k}\left(f_{1}, \cdots, f_{n-1}\right), k=1,2, \cdots$, $n-1$. It is shown that we have here a single-valued analytic transformation (independent of $\Gamma$ ) of the variables $f_{1}, \cdots, f_{n-1}$, having a single-valued analytic inverse. Further, if $\Gamma$ is followed a second time, the next determinations are given by iterating (2). But if a curve $\Gamma^{\prime}$ be used which cannot be deformed into $\Gamma$ in $R$, we shall have a second transformation distinct from (2). In this paper, the idea of the transformation (2) is exploited, and applied to analysis, differential equations, and dynamics. 


\section{Dr. Harry Levy (National Research Fellow): On nets of Tchebycheff in Riemann space.}

The author shows that a necessary and sufficient condition that two space-filling congruences of curves have the property that each forms a system of parallels (in the sense of Levi-Civita) along the other is, first, that the two given congruences generate a space-filling family of surfaces, and, second, that they form nets of Tchebycheff on these surfaces. Further study is made of the case of $p$ congruences of curves enjoying the above properties, and similar results are obtained.

\section{Professor F. D. Murnaghan: A wave-theory formulation} of a geometry of paths. Preliminary communication.

The particular geometry of paths known as Riemannian geometry is visualized by a corpuscular theory of light or matter, but the paths may also be regarded as the extremal curves for the action integral $A=$ $\int p_{1} d q^{\prime}+\cdots+p_{n} d q^{n}-H d t$ in the state space of $2 n+1$ dimensions $(p, q, t)$. The equations of the paths are $a_{r \alpha} x^{\alpha}=0$, where $a_{r s}$ is the curl of the tensor $\left(x_{1}, \cdots, x_{2 n+1}\right) \equiv\left(p_{1}, \cdots, p_{n}, 0, \cdots, 0,-H\right)$. Riemannian geometry in the coordinate space of $n$ dimensions $(q)$ is secured when $H$ is a homogeneous quadratic function of the $p$ 's. It is proposed to introduce a geometry of paths in the $n$-dimensional coordinate, space $(q)$ by integrating the Hamiltonian equations $d q /(\partial H / \partial p)=d p /(-\partial H / \partial q)=d t$ ( $H$ being an arbitrary function of $q, p, t)$ and defining the paths by the equations $q=q\left(q^{0}, p^{0}, t\right)$. By varying the initial values $p^{0}$ we get $\infty^{n-1}$ paths through any point $q^{0}$. It is well known that the Hamiltonian equations define a contact transformation, so that a point $q^{0}$ and the directions $p^{0}$ through it go over into the points $q=q\left(q^{0}, p^{0}, t\right)$ of a spread of $n-1$ dimensions (here $t$ is constant, as are the $q^{0}$, and the ratios of the $p^{0}$ are the variables). This is the wave front which is to be the fundamental element of the proposed geometry, the paths being secondary.

\section{Professor T. R. Hollcroft: Conditions for singularities} of algebraic curves.

Conditions determining singularities of algebraic curves are either metric or projective. Metric conditions determine both the nature and position of a singularity, while projective conditions determine the nature only. Metric and projective conditions do not exist simultaneously for a given singularity. However, when a singularity is defined by metric conditions, the projective conditions that it would have if its position were not given must be considered as latent, since they appear if the singularity is projected to a general position. The number of projective conditions associated with a given singularity is the number of invariant relations among the coefficients of the equation of a curve of any order which are sufficient for the curve to have this singularity. In this paper, the various types of singularities of algebraic curves are built up and the number of projective conditions associated with each singularity is obtained consider- 
ing the curve first as a locus and second as an envelope. Finding the number of projective conditions on the class curve occasioned by a given singularity makes unnecessary the separate consideration of the singularity reciprocal to the one given. Limits are found for the number of certain singularities on curves of given order.

14. Mr. J. H. Neelley: Compound singularities of the plane rational quartic curve. Second paper.

This paper gives a classification of tacnodes and oscnodes, and theorems relating to the duality of these compound singularities. It also considers special quartic surfaces whose plane sections give all types of the plane rational quartic with compound singularities of all kinds.

\section{Professor M. C. Foster: Congruences of lines of special orientation relative to a surface of reference.}

Through every point $M$, of a surface $S$, consider a line $l$ whose direction cosines $\alpha, \beta, \gamma$ relative to the moving trihedral at $M$ are either special functions of the parameters $u$ and $v$, or constant. This paper is concerned with a study of the properties of the rectilinear congruence formed by the lines $l$ of this special orientation.

\section{Professor V. H. Wells: Systems of conics associated with} loci in five-dimensional space.

In this paper the author considers the set of all conics as a five-parameter system, and regards the six coefficients of the equation of such a conic as the homogeneous coordinates of a point in five-dimensional space $S_{5}$, as suggested by Veblen and Young (Projective Geometry, vol. 2, p. 207). The conics of one-, two-, three-, and four-parameter systems are represented in $S_{5}$ by points of $S_{1}, S_{2}, S_{3}$, and $S_{4}$ respectively, where $S_{1}$ is a line, $S_{2}$ a plane, etc. Sub-systems of an $n$-parameter system are represented by loci in the corresponding $n$-space. For example, in a two-parameter system, the sub-systems of degenerate conics and of conics through three points and tangent to one line are represented in $S_{2}$ by a cubic and a conic respectively. The two sub-systems have three degenerate conics in common.

\section{Professor F. A. Foraker: The nepolar reciprocal with respect to a central conic.}

The nepolar line of a point with respect to a central conic is defined to be that straight parallel to the polar line of the point which cuts the diameter through the given point in the point which is the neharmonic conjugate of the given point. The nepolar line of the point $\left(y_{1}, y_{2}, y_{3}\right)$ with respect to the central conic $b_{11} x_{1}{ }^{2}+b_{22} x_{2}{ }^{2}+b_{33} x_{3}{ }^{2}+2 b_{12} x_{1} x_{2}+2 b_{23} x_{1} x_{3}$ $+2 b_{31} x_{3} x_{1}=0$ is the line $\left(b_{11} y_{1}+b_{12} y_{2}+b_{13} y_{3}\right) x_{1}+\left(b_{21} y_{1}+b_{22} y_{2}+b_{23} y_{3}\right) x_{2}$ $+\left(b_{31} y_{1}+b_{32} y_{2}+\left(b_{33}-2 \Delta / \Sigma\right) y_{3}\right) x_{3}=0$, where $\Delta$ and $\Sigma$ have their usual meaning. The nepolar reciprocal of the point $\left(y_{1}, y_{2}, y_{3}\right)$ with respect to the conic $x_{1}{ }^{2}+x_{2}{ }^{2}-x_{3}{ }^{2}=0$ is the line $y_{1} x_{1}+y_{2} x_{2}+y_{3} x_{3}=0$. That of the 
conic $f\left(x_{1}, x_{2}, x_{3}\right)=a_{11} x_{1}{ }^{2}+a_{22} x_{2}{ }^{2}+a_{33} x_{3}{ }^{2}+2 a_{12} x_{1} x_{2}+2 a_{23} x_{2} x_{3}+2 a_{31} x_{3} x_{1}=0$ with respect to the conic $x_{1}^{2}+x_{2}{ }^{2}-x_{3}{ }^{2}=0$ is the conic $f\left(u_{1}, u_{2}, u_{3}\right)=0$.

18. Dr. S. D. Zeldin: Contact transformations in intrinsic geometry.

In this paper the author determines the element of arc, the lowest differential invariant, and the covariant coordinates for each of the following contact groups of the plane: $G_{6}:\left[p, q, x q+q^{\prime}, \frac{1}{2} x^{2} q+x q^{\prime}, x p-y^{\prime} q^{\prime}\right.$, $\left.y^{\prime} p+\frac{1}{2} y^{\prime 2} q\right] ; G_{7}:\left[G_{6}, x p+2 y q+y^{\prime} q^{\prime}\right] ; G_{10}:\left[G_{7},\left(y-x y^{\prime}\right) p-\frac{1}{2} x y^{\prime 2} q, \frac{1}{2} x^{2} p\right.$ $\left.+x y q+y q^{\prime},\left(x y-\frac{1}{2} x^{2} y^{\prime}\right) p+\left(y^{2}-\frac{1}{4} x^{2} y^{\prime 2}\right) q+\left(y y^{\prime}-\frac{1}{2} x y^{\prime 2}\right) q^{\prime}\right]$.

\section{Professor F. W. Beal: Orthogonal systems of curves on} surfaces.

The fundamental equations are expressed in terms of $\alpha_{i}, \beta_{i}, \lambda_{i}$, and $\omega_{i}$, respectively, the first and second curvatures and the derivatives with respect to their parameters of the arcs of the curves of an orthogonal parametric system, and the angles the principal normals of these curves make with the normal to the surface. It is easily shown that if any pair except $\alpha_{1}$ and $\alpha_{2}, \lambda_{1}$ and $\alpha_{2}$, or $\lambda_{2}$ and $\alpha_{1}$ are given functions of the parameters, the general existence theorems apply. In the exceptional cases, the quantities are assumed to be functions of other quantities, and, when desired, of certain derivatives. For example, to show that the parameters $u=c$ and $v=c$ may be congruent helices, it is desirable to let $\partial \omega_{1} / \partial u$ and $\partial \omega_{2} / \partial v$ occur. In certain cases solutions unique to within allowable transformations of parameters are found.

20. Professor W. C. Graustein: On the determination of the linear element of a Riemannian $V_{n}$ from the Christoffel symbols of the second kind.

The author establishes the following theorem for the case $n=2$ : There exists a positive definite quadratic form $g_{a b} d x^{a} d x^{b}$, which has prescribed values for the Christoffel symbols $\left\{a^{c_{b}}\right\}$ of the second kind if, and only if, $\left\{a_{b}{ }_{b}\right\}$ and the Ricci tensor $R_{a b}$, formed from $\left\{a^{c} b\right\}$, are symmetric in $a$ and $b, R_{11} R_{22}-R_{12}{ }^{2}>0$, and the covariant derivative, $R_{a b}, c$, taken with respect to $\left\{a_{b}{ }^{c}\right\}$, is the product of $R_{a b}$ and a tensor of the first order: $R_{a b, c}=X_{c} R_{a b}$; the linear element can then be found by quadratures. Generalizations of the theorem to the case $n=n$ are given for spaces of constant curvature and, more generally, for Einstein spaces.

\section{Professor Dunham Jackson: Some non-linear problems of approximation.}

This paper presents theorems of convergence, of which the following is illustrative. Let $f(x)$ be a given positive function of period $2 \pi$ having a continuous derivative. Among all trigonometric sums of the $n$th order, let $T_{n}(x)$ be one which minimizes the integral of $\left\{f(x)-\left[T_{n}(x)\right]\right\}^{2}$, extended 
over a period. Then $\left[T_{n}(x)\right]^{2}$ converges uniformly toward $f(x)$ as $n$ becomes infinite. A corresponding theorem is obtained if $\left[T_{n}(x)\right]^{2}$ is replaced by $\left[T_{n}(x)\right]^{1 / 2}$. The proofs involve a generalization of Bernstein's theorem on the derivative of a trigonometric sum.

\section{Professor F. D. Murnaghan: On Whittaker's method} for the numerical solution of integral equations.

Whittaker has given (Proceedings of the Royal Society, 1918) a method for the numerical solution of integral equations of Volterra's type based on the approximation to the kernel, which is assumed to be a function of the difference of its arguments, by a sum of $n$ exponential functions of this difference, or by a polynomial in this difference. It is shown in the present paper that the problem is equivalent to the solution of an ordinary linear differential equation of order $n$ with constant coefficients, and Whittaker's formulas follow at once. The Fredholm equation is treated similarly, when the kernel is a function of the difference of its arguments.

\section{Professor R. L. Moore: Abstract sets and the founda-} tions of analysis situs.

Axioms 1, 2, and 4 and Theorem 4 of the author's Foundations of plane analysis situs hold true in euclidean space of any finite number of dimensions. But Axiom 4 does not hold true in a Hilbert space or a space $E_{\omega}$, and Axiom 1 does not hold in the non-separable space $D_{\omega}$. Axiom 2, Theorem 4, and Axiom 1' stated below hold true in all these spaces, and form a basis for a considerable body of theorems, in particular, Theorems $1-10$, and 15 , of the paper cited above. Axiom $1^{\prime}$ : There exists a countable sequence $G_{1}, G_{2}, G_{3}, \cdots$ such that (a) for each $n, G_{n}$ is a collection of regions covering space, (b) if $P_{1}$ and $P_{2}$ are distinct points of a region $R$, there exists an integer $\delta$ such that if $n>\delta$ and $K_{n}$ is a region containing $P_{1}$ and belonging to $G_{n}$, then $K_{n}^{\prime}$ is a subset of $R-P_{2}$, (c) if $R_{1}, R_{2}, R_{3}, \cdots$ is a sequence of regions such that, for each $n, R_{n}$ belongs to $G_{n}$ and such that, for each $n, R_{1}, R_{2}, \cdots, R_{n}$ have a point in common, then there exists a point common to all the point sets $R_{1}^{\prime}, R_{2}^{\prime}, R_{3}^{\prime}, \cdots$.

\section{Professor L. L. Smail: A simplified derivation of the} fundamental properties of double sequences.

In Pringsheim's classical paper on double sequences and series in Mathematische Annalen, vol. 53, reprinted with slight modifications in his Vorlesungen über Zahlen- und Funktionenlehre, and also in London's paper on the same subject in Mathematische Annalen, vol. 53, the derivation of many of the fundamental properties of double sequences is based on a study of the simple sequences contained in the double sequence. By methods explained in the present paper, based on the use of the double sequence only, these fundamental properties may be obtained in a very much simpler way, by use of arguments analogous to those used recently for simple sequences by several writers on the subject of infinite processes. 


\section{Professor W. W. Elliott: Generalized Green's functions for compatible differential systems.}

The theory of Green's functions for incompatible differential systems which consist of a linear differential equation of the $n$th order and $n$ linearly independent boundary conditions involving values of the function and its $n-1$ derivatives at the two end points of a given interval is well known. The existence of generalized Green's functions for certain special types of compatible differential systems has also been shown. In this paper it is shown that the generalized Green's functions for general compatible systems exist, and their characteristic properties and some applications are discussed.

\section{Professor L. L. Silverman: On the omission of terms} in certain summable series.

It is well known that a definition of summability does not in general permit the dropping of a term in a series. In the present paper, a sufficient condition is obtained for the permissibility of dropping a term in the case of a certain class of definitions of summability. This result contains as special cases the results previously obtained by Hurwitz and Silverman, Transactions of this Society, vol. 18 (1917), and by Knopp, Mathematische Zeitschrift, vol. 15 (1922).

27. Professor H. W. Sibert: A new type of singular solution of differential equations of the first order.

The differential equation of the first order and $n$th degree $\Psi(x, y, p)$ $=A_{0} p^{n}+A_{1} p^{n-1}+\cdots+A_{n-1} p+A_{n}=0$, where the $A$ 's are integral functions in $x$ and $y$, may be written $\Psi(x, y, p)=\left(p-\theta_{i}\right)^{2}\left[A_{0} p^{n-2}+B_{3 i} p^{n-3}\right.$ $\left.+\cdots+B_{n-1}, i p+B_{n i}\right]+R_{i}$, where $\theta_{i}(i=1,2, \cdots, n-1)$ is any root of $\Psi_{p}{ }^{\prime}(x, y, p)=0$. It may be shown that the $p$-discriminant $D_{p}(x, y)$ $=C A_{0^{n-1}} \prod_{i=1}^{i=n-1} \Psi\left(x, y, \theta_{i}\right)=C A_{0^{n-1}} \prod_{i=1}^{i=n-1} R_{i}$, and hence any function of $x$ and $y$ which satisfies $\prod_{i=1}^{i=n-1} R_{i}=0$ and $A_{0} p^{n-2}+B_{3 i} p^{n-3}+\cdots+B_{n i}=0$ is a singular solution of $\Psi(x, y, p)=0$ and is not an envelope.

28. Professor H. M. Hosford: On the summability of Fourier-Bessel and Dini expansions.

The object of this paper is to investigate what conditions may be placed upon a function $\phi_{n}(\omega)$ such that the Fourier-Bessel series and the Dini series associated with $f(t)$ will be summable by the following method. If $\lim _{n \rightarrow \infty} \sum_{m=1}^{n} \phi_{n}\left(j_{m}\right) a_{m} J_{\nu}\left(j_{m} x\right) \quad\left[\lim _{n \rightarrow \infty} \sum_{m=1}^{n} \phi_{n}\left(\lambda_{m}\right) b_{m} J_{\nu}\left(\lambda_{m} x\right)\right]$ exists, the Fourier-Bessel [Dini] series will be said to be summable $(\phi)$, and this limit is taken as the sum $(\phi)$ of the series. A method of summability $(\bar{\phi})$ similar to the method $(\phi)$ is given where less stringent restrictions are placed on the function $\phi_{n}(\omega)$ and more stringent restrictions on $f(t)$. The summability theory of both methods is extended to the multiple Fourier-Bessel series and the multiple Dini series. The uniformity of summability by both methods is shown under the appropriate conditions 
of continuity on the function $f(t)$. The method used is essentially a generalization to summability of a method due to Birkhoff for convergence, the tools being Green's function and contour integration.

29. Dr. D. V. Widder (National Research Fellow): $A$ seneralization of Taylor's series.

The series discussed in this paper arises from a particular phase of the problem of the representation of an arbitrary function $f(x)$ in terms of linear combinations of prescribed functions. The function of approximation $s_{n}(x)$ of order $n$ is defined as that linear combination of the first $(n+1)$ of the prescribed functions which has closest contact with $f(x)$ at a given point $x=t$. The series $s_{0}(x)+\sum_{n=1}^{\infty}\left[s_{n}(x)-s_{n-1}(x)\right]$ is then a generalization of Taylor's series to which it reduces if the prescribed functions are $1, x, x^{2}, \cdots$. Conditions for the convergence of the series are discussed. It is found that under certain rather general conditions any analytic function can be expanded in such a series. Examples are given to show that sequences exist possessing the properties imposed.

30. Dr. D. V. Widder (National Research Fellow): On the interpolatory properties of a linear combination of continuous functions.

In his treatment of the mean-value theorems belonging to a linear differential equation, G. Pólya obtained a condition on a set of functions $u_{0}(x), u_{1}(x), \cdots, u_{n}(x)$ which insured that any linear combination of them should enjoy the most important interpolatory properties of a polynomial of degree $n$. The condition imposed presupposes that the functions $u_{i}(x)$ possess continuous derivatives of order $n$. The author obtains an analogous condition, which presupposes only the continuity of $u_{i}(x)$.

31. Dr. D. V. Widder (National Research Fellow): Note on Tchebycheff approximation.

Application is made of the results of the preceding paper to the problem of Tchebycheff approximation. It is found that the condition there imposed on the functions $u_{0}(x), u_{1}(x), \cdots, u_{n}(x)$ is necessary and sufficient that there exist a unique function of approximation in the sense of Tchebycheff to a given continuous function $f(x)$.

32. Dr. Jesse Douglas (National Research Fellow): Reduction of the problem of Plateau to an integral equation.

In this paper the problem of Plateau is formulated so as to require not only a regular simply connected segment $M$ of a minimal surface, bounded by a prescribed contour $\Gamma$, but also a conformal representation of $M$ on a half-plane. $\Gamma$ being defined by the equations $x=x(t), y=y(t), z=z(t)$, the problem is made to depend on the integral equation $(1): \int_{\Gamma}(K(t, \tau) /(\phi(\tau)$ $-\phi(t))) d \tau=0$, where $K(t, \tau)=S x^{\prime}(t) x^{\prime}(\tau), S$ denoting summation over $x, y, z$. This equation is to be solved with a continuous function $\phi$ which takes each real value, including the (signless) infinite value, once and 
only once on $\Gamma$, and which is in general differentiable. The integrand in (1) thus becomes infinite when and only when $\tau=t$. The integral is to be taken in its principal value. The function $\phi$ found, it remains simply to solve three Dirichlet problems for the half-plane, one for each of the coordinates $x, y, z$. This is effected by means of the definite integral corresponding to Poisson's integral for a circular domain. In the important special case where $\Gamma$ is a skew polygon, (1) can be replaced by a certain linear (singular) integral equation.

\section{Professor G. D. Birkhoff: Stability and the equations of dynamics.}

After earlier researches by Laplace, Poisson, and others, on the stability of the solar system, Poincaré proved the complete formal stability of any system leading to equations of Hamiltonian canonical form. (See his Méthodes Nouvelles de la Mécanique Céleste, vol. 2.) The inverse question of the characterization of the differential equations for which such formal stability holds is taken up in the present paper. (See a note by G. D. Birkhoff, Comptes Rendus, August 30,1926.) It is shown that such formal stability is possible if and only if the differential equation results from a Pfaffian variational principle $\delta \int_{t_{0}}^{t_{1}}\left\{P_{1} d x_{1}+\cdots+P_{n} d x_{n}+Q d t\right\}=0$, which can also be transformed into the Hamiltonian form $\delta \int_{t_{0}}^{t_{1}} \eta d \xi_{1}+\cdots$ $\left.+\eta_{m} d \xi_{m}-H d t\right\}=0 \quad(n=2 m)$, by a proper choice of formal variables $\xi_{i}, \eta_{i}$. It is shown that a necessary and sufficient condition that the formal series used in the transformation be convergent is that for a proper selection of the variables $x_{1}, \cdots, x_{n}$, the differential form $P_{1} d x_{1}+\cdots+P_{n} d x_{n}$ becomes an exact differential when half of the variables are annulled. To a certain extent, then, it appears that the property of formal stability alone ensures Pfaffian or Hamiltonian form, and thus constitutes their only characteristic property; also, the Pfaffian form has the advantage of being maintained under the most general point transformation of dependent variables. These results will appear in the author's Chicago Colloquium lectures on Dynamical Systems, soon to appear in book form.

34. Professor Elijah Swift: Canonical forms for ordinary linear differential equations of the second order with periodic coefficients.

This paper considers the ordinary linear differential equation of the second order $y^{\prime \prime}+p(x) y^{\prime}+q(x)=0$, where $p$ and $q$ are periodic functions of $x$, and the two groups of transformations $y=\lambda(x) \bar{y}, x=\psi(\bar{x})$, where $x$ and $y$ are the original variables and $\bar{x}$ and $\bar{y}$ the transformed variables, and where $\lambda$ and $\psi$ are such functions that the coefficients of the transformed equation are periodic with the same period as those of the original equation. By using transformations of the two groups mentioned the equation may be reduced to one of a few canonical forms. If we classify differential equations of the above type using the roots of a certain (algebraic) quadratic equation, we find for the equations of one class that 
the invariants are the ratio of these roots, the number of zeros of a certain solution of our differential equation in a certain interval, and, in some cases the sign of a Wronskian determinant. Any two differential equations with the same invariants may be transformed the one into the other.

35. Dr. C. C. Camp: An expansion associated with a partial differential equation.

The convergence proof for a development in terms of the most general solutions of $\sum_{i=1}^{p}\left(\partial u / \partial x_{i}\right)+(\lambda h+k) u=0$ and the boundary conditions $\left.u]_{x_{i}=-\pi}=u\right]_{x_{i}=\pi}, i=1,2, \cdots, p$, can be effected by an extension of Birkhoff's contour method provided the number of parameters be changed from 1 to $p$. (It is believed that in general this number of parameters is essential to the convergence proof when partial differential equations are involved.) These parameters enter naturally for solutions of the form $u_{1}=\prod_{i=1}^{p} X_{i}\left(x_{i}\right)$. The more general solution $u=e^{-\lambda H-K} u_{1} \phi$ can then be handled with similar Green's functions. Choose $h=D H+\sum_{i=1}^{p} a_{i}\left(x_{i}\right)$, $k=D K+\sum_{i=1}^{p} g_{i}\left(x_{i}\right)$, where $D \equiv \sum_{i=1}^{p}\left(\partial / \partial x_{i}\right)$, and take $a_{i}\left(x_{i}\right)$ either $>0$ or $\equiv 0$, but some $a_{j}>0$; then if $H, K$ and $\phi$ satisfy the boundary conditions, the expansion of $f$ of the usual sort in the form $\sum c^{*} u^{*}$ converges for any interior point to the mean value. The multiple series is to be summed in such a way as to make the transformed principal parameter values together with their sum become infinite.

36. Professor G. C. Evans: Logarithmic potential and convergence in the mean of order less than one.

The author proves that if $u(r, \theta)$ is given by a Poisson-Stieltjes integral it converges in the mean, as $r$ approaches the boundary radius, of all orders less than one. The theorem rests on theorems on convergence in the mean, of order less than one, e.g., if $f_{m}(x)$, not negative, is convergent in the mean of order $\nu$, a subsequence from $f_{m}(x)$ converges to a function $f(x)$ whose $\nu$ th power is summable, and this $f(x)$ is the only function such that the integral of $\left|f-f_{m}\right|$ to the power $\nu$ has the limit zero. The converse statement is obvious. Finally, if $f_{m}(x)$, not negative, approaches zero almost everywhere, and fails to converge in the mean, of order $\nu$, then there will be a subsequence of these functions the integral of whose $\kappa$ th powers will become infinite, if $\kappa>\nu$.

\section{Dr. S. Mandelbrojt: On the behavior of functions} developable in Dirichlet series.

The author considers the conditions on functions represented by Dirichlet series $f(s)=\sum a_{n} e^{-\lambda_{n} s}, s=\sigma+i t$, necessary and sufficient for absolute convergence. Such conditions show that the functions so developable form a very special class, possessing, if the coefficients are real, a certain symmetry in the distribution of values for the line $\sigma=\sigma^{\prime}$. Let $R$ be the upper bound of $|f(s)|$ on $\sigma=\sigma^{\prime}$. The measures of the sets of points on $\sigma=\sigma^{\prime}$ where $f(s)$ takes on values near given values $e^{i \phi} R$ and $e^{i(\phi+\pi)} R$, 
respectively, differ asymptotically little. More precise formulas are given, in the direction of earlier results stated in the Comptes Rendus, August 2, 1926 , which hold only when $\lambda_{n}$ takes the form $\log p_{n}, p_{n}$ being the $n$th prime.

38. Professor Tomlinson Fort: An elementary proof by mathematical induction of the equivalence of the Cesàro and Hölder sum formulas.

The author gives an explicit formula for the Cesàro sum to $n$ in terms of the Hölder sum to $n$, and vice versa. These formulas are readily proved by mathematical induction, and from them, in case of the existence of one limit, the existence and equality of the other follows almost by inspection.

39. Mr. Mark Kormes: Concerning a property of nowhere dense, perfect sets.

In this note the following theorem is proved: Given a perfect, nowhere dense, linear set $\mathfrak{B}$ and let $P$ be its kernel of equal measure. The set of points $R$, where the density is undetermined, is of the second category with respect to $P$.

40. Professor R. G. Putnam: On elements of accumulation and neighborhoods in the theory of abstract sets.

This paper compares the neighborhood conditions resulting from the four conditions of F. Riesz and also a fifth condition with the Hausdorff neighborhood axioms. It is shown in particular, if the four Riesz conditions are satisfied, that corresponding to any pair of distinct elements of the class there exists at least one pair of disjoint neighborhoods.

\section{Professor Henry Blumberg: On the generalization of} the theorem that a perfect set has the cardinal number $c$.

An extension of this theorem concerning sets of points to sets of sets may be made by means of the notion of distance between sets (Hausdorff's "Entfernung") or, in case of components of a compact closed set, by means of the notion of $\rho$-connectedness. The latter method exhibits the theorem in question and R. L. Moore's extension of it (Proceedings of the National Academy, May, 1924), as derivable from a common source.

\section{Professor Henry Blumberg: A theorem on arbitrary} real functions of two variables with an application to derivatives.

Let $f(x, y)$ be any real function of two variables defined for the entire $x y$-plane, and $l$ any straight line of this plane. By the interval of approach $I_{P d}$ we understand the interval $(i, s)$ where $i$ is $\lim \inf f(x, y)$ as $(x, y)$ approaches the point $P$ (of $l$ ) along the direction $d$, and $s$ equals the corresponding lim sup. If now $d_{0}$ is any fixed direction, there exists an enumerable set $E_{d_{0}}$ such that if $P$ is any point of $l$ not in $E_{d_{0}}$, then all the intervals of approach $I_{P d}$, for all possible directions from the same side of $l$ as that 
of $d_{0}$, overlap, or at least abut, $I_{P d_{0}}$. If $\left\{d_{n}\right\}$ is a sequence of directions of approach, all from one and the same side of $l$, there exists an enumerable set $E_{\left\{d_{n}\right\}}$ such that if $P$ is any point of $l$ not in $E_{\left\{d_{n}\right\}}$, then not only do the intervals $I_{P d_{n}}$ all mutually overlap or abut, but every $I_{P d}$ where $d$ is arbitrary but from the same side of $l$ as the $d_{n}$, overlaps or abuts every $I_{P d_{n}}$. As a particular application to functions of one variable, we have the familiar result that $D^{+} \geqq D_{-}$except at most at $\boldsymbol{\aleph}_{0}$ points.

\section{Professor Henry Blumberg: Note concerning closed sets.}

The proof that certain point sets, like the derived set, the set of condensation points, or the set of points of inexhaustibility, are closed is simple enough as usually presented, but a separate effort of thought is expected in every new case. It is true, however, that if a point set $S$ is defined as the aggregate of points every neighborhood of which has a given neighborhood property, then $S$ is closed; and conversely, every closed set is definable as the set of points every neighborhood of which has a certain neighborhood property. This fact makes obvious, from the definition of the above-mentioned or similar sets, that they are closed.

\section{Professor R. L. Wilder: Proof of the non-existence of a certain type of regular point set.}

The author has shown in another connection that a regular connected point set which contains more than one point and possesses the property of remaining connected upon the omission of any connected subset is a simple closed curve. Hence there does not exist an unbounded regular connected set which has this property. The present paper takes up the case of an unbounded regular connected point set which remains connected upon the omission of any bounded connected subset. It is shown that such a set does not exist. For the more special case of closed sets, this was proved by J. R. Kline (Fundamenta Mathematicae, vol. 5 (1924), pp. 3-10).

\section{Dr. H. M. Gehman: Note on Schoenflies' definition of a continuous curve.}

According to Schoenflies' definition, in order that a bounded continuum be a continuous curve, it is necessary and sufficient that the remainder of space have certain properties. R. L. Moore has shown that these properties are neither necessary nor sufficient if the space be of more than two dimensions. In this paper, it is shown that Schoenflies' properties are necessary, but not sufficient, if the space be itself a continuous curve in the plane.

\section{Professor H. S. Vandiver: Transformations of the Kummer criteria in connection with Fermat's last theorem. Second paper.}

This paper continues the investigations of the author's first paper with this title (Annals of Mathematics, (2), vol. 27 (1926), pp. 171-176), and 
transformations are obtained which will be used later in the derivation of the criteria $m^{p-1} \equiv 1\left(\bmod p^{2}\right)$ for small values of $m$, with respect to the first case of Fermat's last theorem.

\section{Professor Olive C. Hazlett: On the composition of}

\section{polynomials.}

This paper considers the problem of the determination of all homogeneous polynomials $f(x)$ in $n$ variables, $x_{1}, x_{2}, \cdots, x_{n}$, such that $f(x) f(\xi)=f(X)$, where the X's are bilinear in the $x$ 's and $\xi$ 's. If the field of definition is the field of complex numbers, then it appears that there is no such $f(x)$ which is irreducible of degree $n$. Denote by $\Delta(x)$ and $\Delta^{\prime}(x)$ the first and second determinants of the general number $x_{1} e_{1}+x_{2} e_{2}+\cdots+x_{n} e_{n}$ of the linear algebra defined by the composition. If $f(x)$ cannot be expressed in less than $n$ variables, then the irreducible factors of $f(x)$ coincide with the irreducible factors of $\Delta(x)$ and $\Delta^{\prime}(x)$. From this theorem are proved some general properties of homogeneous polynomials that admit a composition.

48. Professor Olive C. Hazlett: Note on formal modular invariants.

This paper gives a second proof of the finiteness theorem for formal modular invariants and covariants of a system of binary forms with respect to the Galois field $G F\left[p^{n}\right]$, of order $p^{n}$.

\section{Professor Olive C. Hazlett: Ideals for any linear associative algebra.}

This paper defines an ideal for a general linear associative algebra $A$ of order $n$ over the field of rational numbers as a set of numbers belonging to an arithmetic of $A$ which is closed under addition and subtraction and under multiplication (on right or left or both) by any integer of this arithmetic. Then it is proved that, for a semi-simple algebra, every ideal has a finite basis of order $n$ and that if $A$ is the direct sum of two algebras $B$ and $C$ then we know the ideals of $A$ as soon as we know those of $B$ and $C$ and conversely. Moreover, a knowledge of the factorization of the ideals of $A$ is equivalent to a knowledge of the factorization of the ideals of $B$ and of $C$, and thus the study of ideals of a semi-simple algebra is reduced to that of simple algebras. Likewise, a study of ideals of a simple algebra is reduced to that for a division algebra. By using a generalization of Kronecker's fundamental theorem, it is proved that if $\alpha \neq 0$ is any ideal of a division algebra, then there exists an ideal $\beta$ such that $\alpha \beta$ is a principal scalar ideal $(q)$ and such that $\beta \alpha=(q)$, which we call the norm of $\alpha$. From this theorem flow the theorems about the uniqueness of factorization into prime ideals, which are closely analogous to those for the ideals defined for an algebraic field.

50. Professor James Pierpont: A generalization of the secular equation.

This paper will appear in full in an early issue of this Bulletin. 
51. Professor Harris Hancock: An extremely simple proof of a fundamental theorem in ideals.

In the Dedekind theory of moduls, it is shown that if $\mathfrak{a}$ and $\mathfrak{b}$ are any two integral moduls and if $\mathfrak{a}$ is contained in $\mathfrak{b}$ (written $\mathfrak{a}>\mathfrak{b}$ ), then $\mathfrak{a} / \mathfrak{b}=\mathfrak{i}$, where $i$ is an integral modul. If the discussion has to do with integral ideals, which are a special kind of modul, and if $T$ is any algebraic integer that is divisible by the ideal $\mathfrak{a}$, then $\mathfrak{D} T / \mathfrak{a}=\mathfrak{b}$, where $\mathfrak{b}$ is an integral ideal, and where $\mathfrak{D}$ is the order-modul ("Ordnung" or "Art") as defined and used by Dedekind. It follows that $\mathfrak{a} \mathfrak{b}=\mathfrak{a}(\mathfrak{D} T / \mathfrak{a})=(\mathfrak{a} / \mathfrak{a}) \mathfrak{D} T=\mathfrak{D} T=\mathfrak{D} T$. That is, associated with every ideal $\mathfrak{a}$ there is another integral ideal $\mathfrak{b}$ of the same realm such that the product $\mathfrak{a} \mathfrak{b}$ is a principal ideal. With this it is proved (see Bachmann, Allgemeine Arithmetik der Zahlenkörper, p. 168) that if $\mathfrak{i}$ is contained in $\mathfrak{a}$, then $\mathfrak{i}=\mathfrak{a} \mathfrak{g}$, where $\mathfrak{g}$ is an integral ideal.

52. Professor Philip Franklin: A theorem of Frobenius on quadratic forms.

For a regularly arranged quadratic form, i.e., one in which no two consecutive principal minors vanish, the signature of the form may be determined from the signs of these minors. In certain cases where consecutive minors vanish, the result still holds, as Frobenius has shown. In this paper a simplified proof of this result is given.

\section{Professor O. E. Glenn: A list of differential combinants.}

Combinants of pairs of binary differential quantics are expressions in certain determinants whose elements are derivatives. In this paper, thirty cases for forms of low orders are tabulated in Maschke's symbolic notation, some being expressed also in the actual form.

\section{Professor Harris Hancock: Equivalent (quadratic)} ideals have similar lattice points.

The proof for the general realm $\Re\left(m^{1 / 2}\right)$ is the same as that for a particular realm, which for convenience is taken as $\Re\left(i 5^{1 / 2}\right)$. The canonical form of ideals of this realm is $\mathfrak{i}=\left(a, b+i 5^{1 / 2}\right)$, where $a$ and $b$ are rational integers and $a>b$. (Sommer, Vorlesungen über Zahlentheorie, pp. $207 \mathrm{ff}$.) If $\mathfrak{i}$ is equivalent to $\mathfrak{i}_{1}=\left(A, B+i 5^{1 / 2}\right), A, B<A$ rational integers, then there exist two algebraic integers $\alpha=c+i 5^{1 / 2} d$ and $\alpha_{1}=C+i 5^{1 / 2} D, a, b, C, D$ rational integers, such that $(\alpha) \hat{i}=\left(\alpha_{1}\right) \mathfrak{i}_{1}$. This relation gives, if

$$
\mathfrak{i}=a x+b y+i 5^{1 / 2} y, \quad \text { and } \mathfrak{i}_{1}=A X+B Y+i 5^{1 / 2} Y \text {, }
$$

the following equations:

$c a x+(c b-5 d) y=C A X+(C B-5 D) Y, d a x+(b d+c) y=D A X+(B D+C) Y$. From these two equations, express $x$ and $y$ in terms of $X$ and $Y$. Due to the fact that $N(x) N(\mathfrak{i})=N\left(x_{1}\right) N\left(\mathfrak{i}_{1}\right)$, it is seen that the determinant of the two expressions for $x$ and $y$ is unity. Reversing the process, and interchanging capitals and small letters, it is seen that the determinant for the corresponding expressions for $X$ and $Y$ is unity. 


\section{Professor I. J. Schwatt: The sum of certain types of Fourier series.}

The author has developed methods by which the sum of types of Fourier series like $\sum_{n=1}^{\infty}( \pm 1)^{n-1}\left(\sin ^{p}(b+n \theta)\right) /(a+n h)$, and $\sum_{n=1}^{\infty}( \pm 1)^{n-1}$ $\left(\begin{array}{c}m+n-1 \\ n-1\end{array}\right)\left(\sin ^{p}(b+n \theta)\right) /(a+n h)$, can be obtained. The methods and the results are believed to be new.

\section{Professor J. L. Walsh: On the expansion of a harmonic} function in terms of harmonic polynomials.

A necessary and sufficient condition that a finite region $R$ of the plane have the property that every function harmonic interior to $R$ and continuous in the corresponding closed region be uniformly expansible in this closed region in a series of harmonic polynomials is that the boundary of $R$ be likewise the boundary of an infinite region.

\section{Professor C. N. Reynolds: On the problem of coloring} maps in four colors. Second paper.

This paper is a continuation of the author's earlier paper on this subject (Annals of Mathematics, (2), vol. 28, pp. 1-15), in which he developed analytically some of the implications of the known theorems concerning irreducible maps, i.e., maps of simply connected closed surfaces which, while requiring five colors, have as few regions as any other map requiring five colors. In the present paper the author applies to the non-pentagons of an irreducible map the methods which he previously applied to the pentagons of such a map. Corresponding to the proposition that an irreducible map must contain at least one pentagon in contact with at least two regions of fewer than seven sides each (proved by Franklin) there follows, as a corollary of a theorem of this paper, the existence, in an irreducible map, of at least twelve pentagons of this type. Other known propositions are strengthened and new propositions established.

\section{Professor Marston Morse: The type number and rank of a closed extremal and a consequent theory in the large.}

In the ordinary regular form of the calculus of variations theory, let there be given a periodic extremal $g$. Let $g$ be cut across by $n$ arcs which, taken in circular order, are set nearer together than any two successive conjugate points on $g$. Let a closed broken extremal be formed with successive vertices on these successive arcs. The value of the given integral taken along this broken extremal will be a function, say $f$, of the $n$ distances of the $n$ vertices from a given side of $g$. The author shows that the matrix a of the symmetric quadratic form $Q$ making up the terms of the second order of $f$ will be of rank $n, n-1$, or $n-2$, respectively, according as the Jacobi differential equation set up for $g$ has (i) no non-zero periodic solution, (ii) a one-parameter family of periodic solutions and no more, or (iii) only periodic solutions. The author means by the "type number of $g$ " the "index of inertia" of $-Q$, and this number is determined in terms of the number of 
successive conjugate points of $g$ and another invariant. These results, in the small, taken with the author's "relations between critical points," lead to a theory in the large.

59. Dr. C. H. Langford: Theorems on deducibility. Second paper.

Questions of deducibility arise especially in connection with sets of defining properties for types of order. A set of properties $p, \cdots, s$ may be related to a property $q$ in such a way that $q$ follows from $p, \cdots, s$ jointly, or in such a way that $q$ is false follows from $p, \cdots, s$ jointly, or in such a way that neither $q$ nor $q$ is false follows from $p, \cdots, s$; and similarly, a class of properties $Q$ may be such that if $q$ be any one of these properties, then either $q$ or $q$ is false follows from $p, \cdots, s$, or $Q$ may be such that for at least one property neither that property nor its contradictory follows from $p, \cdots, s$. This paper is concerned with $Q$ as the class of all firstorder functions on the base $K, R_{2}$, and with $p, \cdots, s$ as a set of defining properties for discrete series with a first but no last element and with but one element having no immediate predecessor. For any first-order function $q$ on $K, R_{2}, q$ follows from the set or $q$ is false follows from the set.

\section{Professor Philip Franklin: The canonical form of one- parameter groups.}

The author deals with a single Lie one-parameter group, and uses the canonical form to find the $n$th extended group, and the differential equation of the $n$th order invariant under the group. This method leads to the tables of known classes of solvable groups much more directly than the methods customarily used. Second-order equations invariant under two groups are next considered. It is shown that a pair of infinitesimal transformations can not always be reduced to canonical form by quadratures (contradicting Theorem 3, Annals of Mathematics, (2), vol. 25, p. 363) but that if we know a differential equation of the second order which is invariant under them, we can so reduce them by quadratures. This explains an apparent paradox in Lie-Scheffers, Differentialgleichungen, pp. 424-457.

\section{Professor B. H. Camp: Note on the correlation between} two frequencies.

A well known theorem in the theory of sampling gives the coefficient of correlation between the frequencies with which two given variables will occur in a sample as $r_{i j}=-\left(p_{i} p_{j} / q_{i} q_{j}\right)^{1 / 2}$, where $p_{i}=1-q_{i}$, and $p_{j}=1-q_{j}$ are the relative frequencies with which these variables are found in the infinite universe from which the sample is drawn. Pearson's proof (Biometrika, vol. 2 (1902-03), pp. 273-81) makes an explicit assumption which, though seemingly reasonable and actually correct, can as well be avoided, and then subsequently derived as a corollary if the method of proof is based on the equation of the frequency surface to which this coefficient of correlation applies. This surface has certain other interesting characteristics. 
62. Professor James Pierpont: Optics in hyperbolic space.

The author treats some of the more elementary problems, assuming the rays of light to obey Fermat's law $\delta \int n d s=0$. Reflection and refraction on plane and spherical surfaces and in prisms are treated. It is shown that the theory of central optical collineation, so useful in euclidean space, does not hold. Finally, Bouguer's theorem is extended to this space. The method employed applies also to elliptic space. In a second paper some of the more advanced parts of optics will be discussed.

63. Professor H. S. Vandiver: Applications of algebraic number theory to congruences involving binomial coefficients.

In this paper the theory of power characters in an algebraic field is used to obtain new theorems concerning congruences involving binomial coefficients, including the following result: If $p$ and $l$ are odd primes; $p$ belongs to the even exponent $f$ modulo $l ; p^{f}-1=c l$, then

$$
\left(\begin{array}{c}
c \\
i
\end{array}\right)+\left(\begin{array}{c}
c \\
i+l
\end{array}\right)+\left(\begin{array}{c}
c \\
i+2 l
\end{array}\right)+\cdots
$$

is divisible by $p$ for $i=0,1, \cdots, l-1$, with the exception of $i=k$, where $k$ is the least positive residue of $c / 2$, modulo $l$, and for $i=k$ is congruent to 1 , modulo $p$.

\section{Professor H. F. MacNeish: Theorems concerning spheres associated with a tetrahedron generalized to space of $n$ dimensions.}

For any tetrahedron $A_{1} A_{2} A_{3} A_{4}$ there is an inscribed sphere with center $O$, four escribed spheres with centers $\mathrm{O}_{1} \mathrm{O}_{2} \mathrm{O}_{3} \mathrm{O}_{4}$, and three exscribed spheres tangent to the four faces externally with centers $O_{12}, O_{13}, O_{14}$. We have the following theorems. Theorem I: The linear polar planes of $O, O_{12}, O_{13}$, and $\mathrm{O}_{14}$ as to the given tetrahedron and as to the tetrahedron $\mathrm{O}_{1} \mathrm{O}_{2} \mathrm{O}_{3} \mathrm{O}_{4}$ coincide, and the three points of the set $O, O_{12}, O_{13}, O_{14}$ not chosen as the pole are the vertices of the diagonal triangle of the complete quadrilateral section of the two tetrahedrons in the linear polar plane. Theorem II: The three tetrahedrons $A_{1} A_{2} A_{3} A_{4}, O_{1} O_{2} O_{3} O_{4}$, and $O O_{12} O_{13} O_{14}$ are perspective in pairs in four ways from the vertices of the third tetrahedron. Theorem $I$ is generalized to space of odd dimension $2 n+1$.

65. Professor J. F. Ritt and Mr. Eli Gourin: An assemblage-theoretic proof of the existence of transcendentally transcendental functions.

Th's paper appears in full in the present issue of this Bulletin.

$$
\text { R. G. D. RichaRdSON, }
$$

Secretary. 